\title{
Criminologie
}

\section{La probation pour mineurs au Québec}

\section{Marc LeBlanc}

Volume 8, numéro 1-2, 1975

Délinquance juvénile au Québec

URI : https://id.erudit.org/iderudit/017040ar

DOI : https://doi.org/10.7202/017040ar

Aller au sommaire du numéro

Éditeur(s)

Les Presses de l'Université de Montréal

ISSN

0316-0041 (imprimé)

1492-1367 (numérique)

Découvrir la revue

Citer cet article

LeBlanc, M. (1975). La probation pour mineurs au Québec. Criminologie, 8(1-2), 101-118. https://doi.org/10.7202/017040ar d'utilisation que vous pouvez consulter en ligne.

https://apropos.erudit.org/fr/usagers/politique-dutilisation/ 
LA PROBATION POUR MINEURS AU QUÉBEC

Marc LeBlanc 
Depuis quelques années, la probation pour mineurs est sujet à controverse. Tous ceux qui ceuvrent dans le système de la justice pour mineurs remettent en cause soit son insertion dans les structures, soit ses opérations, soit son efficacité ou tout à la fois. Une telle mise en question s'est souvent faite sans des données statistiques élémentaires sur la clientèle, le personnel et les opérations à l'intérieur du Service de probation. Cet article veut combler ce vide en rapportant les résultats des recherches qui se sont intéressées à la probation durant les dernières années.

En 1918, on institua à Montréal le premier tribunal pour mineurs et on y nomma des agents de surveillance, ancêtres des agents de probation. Mais ce n'est qu'en 1958 que fut institutionnalisé le Service de probation à Montréal par la nomination d'un directeur. Pour l'ensemble du Québec, c'est en 1964 que le directeur provincial fut nommé. Depuis, il y a des agents de probation dans tous les districts judiciaires et ceux-ci appartiennent à 14 services locaux de probation.

Administrativement le Service de probation est indépendant des Cours de Bien-Être social ; organiquement il y est rattaché en plus de la dépendance envers le ministère des Affaires sociales. Le Service de probation, selon la démonstration de Lippé (1969), est à la fois un système social avec ses normes relationnelles et régulatoires, ses rôles... et une organisation bureaucratique avec sa hiérarchie, ses spécialisations...

On reconnaît un ensemble varié et complexe de rôle à l'agent de probation. En effet, Bouffard (1969) en analysant les textes légaux, a identifié les rôles suivants : faire l'enquête deman- 
dée par le juge, conseiller le tribunal et assurer une surveillance et une intervention en vue d'une réadaptation sociale.

Pour terminer cette présentation générale du Service de probation, il convient de mentionner que depuis quelques années ce service opère dans un contexte d'incertitude. En effet, on parle de faire passer la tutelle du service au ministère de la Justice et de l'intégrer à la probation adulte; on propose un service de protection de la jeunesse; on parle d'intégrer la probation aux Centres de services sociaux (C.S.S.) ; avec la création des Centres de services sociaux (C.S.S.) et de leur comité d'admission aux Centres d'accueil, de même qu'avec la mise en place des Centres locaux de services sociaux (C.L.S.S.) la pratique et l'acheminement des cas risquent d'être bouleversés; on engage de plus en plus de personnel avec une formation universitaire ; on parle de sectorisation ; ... à mesure que se rationalise et s'organise l'application des politiques sociales et judiciaires, les interrogations se font de plus en plus pressantes et l'agent de probation travaille dans un climat d'anxiété.

À la suite de ces commentaires sur l'organisation du Service de probation et le climat qui y règne, nous dresserons un portrait statistique de sa clientèle, de son personnel et de ses operations.

\section{A. LA CLIENTĖLE}

Le case-load du Service de probation pour mineurs du Québec comptait, à la fin de 1971 et au début 1972, 5269 cas actifs. Dans cette section, nous décrirons ces cas sur les plans de l'âge, du sexe, de l'occupation et selon leurs situations familiale et légale. Pour terminer, nous présenterons une typologie de la clientèle.

3841 clients $(73,01 \%)$ du Service étaient de sexe masculin par rapport à $1420(26,99 \%)$ de sexe féminin. Si les trois quarts des enfants étaient de sexe masculin ils avaient en moyenne 14,93 ans avec une répartition bi-modale à 16 et 17 ans. $4,66 \%$ des clients ont 9 ans et moins; il y en a $14,36 \%$ entre 10 et 13 ans, $29,02 \%$ de 14 et 15 ans, $51,96 \%$ de 16 ans et plus, dont $6,43 \%$ de 18 et 19 ans. Done la majorité se situe dans les catégories d'âge de 16 et 17 ans. La très grande majorité des clients fréquentent l'école; en effet, $70,78 \%$ (3 672) sont des écoliers par rapport à 13,43\% (697) qui travaillent et à $15,79 \%$ (819) qui sont oisifs. 
$54,75 \%$ (2 868) des clients possèdent une famille intacte, c'est-à-dire composée d'un père et d'une mère résidant ensemble. $11,49 \%(602)$ des clients ont perdu un de leurs parents par décès et $33,75 \%$ (1768) des clients n'ont pas des parents résidant ensemble.

Par ailleurs, il convient de noter qu'une mineure aura une probabilité plus grande d'avoir une famille non intacte et il en est de même si le client est un enfant ou un jeune adolescent (LeBlanc, 1972).

Quelle est l'étiquette légale des clients ? Ont-ils des antécédents à la Cour? En raison de quels motifs ont-ils été référés à la Cour ? Des 5269 clients, 2979 sont classés article $20(56,54 \%)$ et 2290 sont étiquetés article $15(43,46 \%)$. Parmi la clientèle du Service 62,30\% (3 089) n'ont pas d'antécédents à la Cour ; $8,96 \%$ (444) ont déjà comparu sous un article $15,24,77 \%$ (1 228) sous un article 20 et $3,97 \%$ (197) sous un article 15 et un article 20.

Le tableau 1 rapporte la distribution de la clientèle suivant le motif de référence au Service de probation.

Il ressort que le vol est nettement le type de délit le plus fréquent et il dépasse de loin les autres types de délits avec $35 \%$ d'entre eux. L'agression est le type de délit le moins fréquent avec $2,97 \%$. La fugue compte pour $12,51 \%$ et les autres types de

TABLEAU 1

Motifs de référence

\begin{tabular}{lrrrr}
\hline \multicolumn{1}{c}{ Types } & $\mathrm{N}$ & $\begin{array}{c}\% \\
\text { sur } \\
\text { ensemble }\end{array}$ & $\begin{array}{c}\% \\
\text { sur les } \\
\text { délits }\end{array}$ & $\begin{array}{c}\% \\
\text { sur les } \\
\text { autres motifs }\end{array}$ \\
\hline Agression & 100 & $1,95 \%$ & $2,97 \%$ & - \\
Vol & 1234 & $24,06 \%$ & $36,75 \%$ & - \\
Vol qualifié & 636 & $12,40 \%$ & $18,94 \%$ & - \\
Communautaire & 240 & $4,68 \%$ & $7,14 \%$ & - \\
Sexuelle & 174 & $3,39 \%$ & $5,18 \%$ & - \\
Automobile & 214 & $4,17 \%$ & $6,37 \%$ & - \\
Fugue & 420 & $8,19 \%$ & $12,51 \%$ & - \\
Drogue & 185 & $3,61 \%$ & $5,51 \%$ & - \\
Vandalisme & 154 & $3 \%$ & $4,58 \%$ & - \\
Foyer problème & 1003 & $19,56 \%$ & - & $56,63 \%$ \\
Troubles de & 768 & $14,98 \%$ & - & $43,37 \%$ \\
comportement & $\mathbf{7 6 8}$ & $100 \%$ & $100 \%$ & $100 \%$ \\
\hline Total & 5128 & &
\end{tabular}


délits pour de 4 à $7 \%$ de l'ensemble des délits. Il est intéressant de noter que parmi les autres motifs de référence, il y a $57 \%$ de cas de foyers problèmes et $43 \%$ de cas de troubles de comportement. Finalement, il nous faut noter que, si l'on calcule les pourcentages que représentent tous les types de motifs de référence, le vol domine avec $24,06 \%$ suivi des motifs relatifs aux troubles de comportements et aux foyers problèmes avec $15 \%$; les sept autres types comptant pour moins de $15 \%$.

Après avoir décrit la clientèle sur les plans de leurs caractéristiques personnelles et légales, il convient de rapporter quelques conclusions de LeBlanc (1972) sur les relations entre elles.

Il ressort que les clients de sexe masculin ont plus d'antécédents à la Cour que ceux de sexe féminin, $82 \%$ contre $55 \%$. Que plus le client est âgé plus il a une probabilité élevée d'avoir des antécédents à la Cour : $8 \%$ entre 1 et 9 ans, 24\% entre 10 et 13 ans, $38 \%$ à 14 et 15 ans et $44 \%$ à partir de 16 ans. De plus, le client fréquentant l'école aura moins souvent des antécédents à la Cour ; $66 \%$ n'ont pas d'antécédents contre $54 \%$ chez les travailleurs et les oisifs.

Finalement, les clients dont les parents ne demeurent pas ensemble ont une proportion légèrement plus importante de cas sans antécédents, $66 \%$ contre $60 \%$ lorsqu'un parent est décédé ou si les parents demeurent ensemble.

Le motif de référence variera-t-il, lui aussi, suivant les caractéristiques des clients ? Oui, car les motifs de référence diffèrent selon le sexe, l'âge, l'occupation du client et l'état de sa famille. En effet, le garçon sera référé pour vol $(31 \%)$, pour vol qualifié $(17 \%)$, en raison d'un foyer problème $(14 \%)$ et de troubles de comportement (13\%); par contre, les filles seront référées en raison d'un foyer problème (34\%), d'une fugue (23\%) et de troubles de comportement $(21 \%)$. Donc, il y a une variation de la nature et de l'importance du motif de référence suivant le sexe. Il en est de même pour l'âge car chez les clients de 1 à 9 ans, $90 \%$ sont référés en raison d'un foyer problème; chez les 10 à 13 ans, on trouve dans l'ordre : foyer problème (39\%), troubles de comportement $(20 \%)$ et vol $(19 \%)$. C'est à partir de 14 et 15 ans que les variations s'accentuent car le vol représente $25 \%$, les troubles de comportement $17 \%$, les foyers problèmes $16 \%$ et le vol qualifié $13 \%$; et à partir de 16 ans le vol compte pour $27 \%$, le vol qualifié pour $15 \%$ et les troubles de 
comportement pour $13 \%$ et la fugue comme le foyer problème marquent $10 \%$.

Pour sa part, l'occupation est associée à la nature et à l'importance du motif de référence. Chez les écoliers, $24 \%$ volent, $22 \%$ sont issus de foyers problèmes, $17 \%$ ont des troubles de comportement et $11 \%$ font des vols qualifiés. Chez les travailleurs $30 \%$ volent, $10 \%$ font des vols qualifiés et $10 \%$ fuguent. Finalement, les oisifs volent dans $22 \%$ des cas, ont des foyers problème (17\%), font des vols qualifiés $(13 \%)$, ont des troubles de comportement $(12 \%)$ et fuguent $(10 \%)$.

Quant à la relation entre l'état de la famille et le motif de référence on note, que lorsque les parents résident ensemble, le vol (249) domine, suivi du vol qualifié (15\%) et des troubles de comportement (13\%). Par contre si les parents ne résident pas ensemble, les motifs de référence sont : le foyer problème $(35 \%)$, le trouble de comportement $(16 \%)$ et le vol $(16 \%)$. Finalement si un des parents est décédé les motifs de référence prédominants sont dans l'ordre : vol (24\%), troubles de comportement $(20 \%)$, foyer problème $(19 \%)$ et vol qualifié $(10 \%)$.

$\mathrm{Si}$ nous considérons maintenant l'étiquette légale, nous notons que les articles 20 sont l'apanage des garçons et les articles 15 celui des filles; en effet, il y a $69 \%$ d'article 20 chez les premiers et $77 \%$ d'article $15 \mathrm{chez}$ les secondes. Les articles 15 diminuent avec l'âge ; ils passent de $98 \%$ entre 1 et 9 ans, à $66 \%$ entre 10 et 13 ans, à $43 \%$ à 14 et 15 ans et à $32 \%$ à 16 ans et plus. Par ailleurs, quelle que soit l'occupation du client il y a une proportion plus importante d'articles $20 ; 74 \%$ chez les travailleurs, $59 \%$ chez les oisifs et $53 \%$ chez les écoliers. Finalement, l'état de la famille semble influencer le choix de l'étiquette légale : les articles 20 comptent pour $68 \%$ lorsque les parents demeurent ensemble, $52 \%$ si un des parents est décédé et $40 \%$ lorsque les parents ne demeurent pas ensemble.

De façon à identifier les types de clients, neuf variables ont été traitées à l'aide de l'analyse d'association par attributs dichotomiques. Cette technique statistique permet de grouper les variables de façon à constituer des classes homogènes d'individus. Pour ce faire on choisit la variable qui démontre le plus d'association avec les autres et l'on répète l'opération dans chacun des groupes constitués successivement (Le Blanc, 1971). Les variables qui ont été utilisées sont les suivantes : 
Sexe : masculin - féminin

Age : 1 à 15 ans -16 et plus

Parents : demeurent ensemble - ne demeurent pas ensemble Occupation : écolier - oisif ou travailleur

Article : $20-15$

Antécédents à la Cour : oui - non

Nature du cas : enfant en danger - autres cas

Délinquance : traditionnelle (vol-agression) — autre délinquance

Délinquance nouvelle (sexe-drogue...) - autre délinquance.

Les types de clients se définissent d'une façon générale à l'aide de 4 variables et on peut noter l'existence de 19 types de clients différents au niveau de la probation juvénile. Les types les plus importants comptent $12 \%$ de la clientèle et les moins nombreux $1 \%$ des sujets.

Il y a neuf types (A à I) qui concernent les articles 20 et 10 types (J à S) touchant les articles 15 . Par la suite, la variable prédominante est le motif de référence ou le type de délinquance. Le tableau 2 décrit donc en détail les types de clients identifiés et la figure 1 permet de les visualiser.

La lecture des types de clients du Service de Probation nous permet de conclure à l'existence de deux clientèles : les cas délin-

TABLEAU 2

Types de clients

\begin{tabular}{|c|c|c|c|}
\hline Type & Caractéristiques du type & $\begin{array}{l}\text { Nombre } \\
\text { de sujets }\end{array}$ & $\%$ \\
\hline A & $\begin{array}{l}\text { Un article } 20 \text { ayant commis une délin- } \\
\text { quance traditionnelle entre } 1 \text { et } 15 \text { ans } \\
\text { et avec des antécédents à la Cour. }\end{array}$ & 353 & $6,69 \%$ \\
\hline B & $\begin{array}{l}\text { Un article } 20 \text { ayant commis une délin- } \\
\text { quance traditionnelle entre } 1 \text { et } 15 \text { ans } \\
\text { et sans antécédents à la Cour. }\end{array}$ & 399 & $7,57 \%$ \\
\hline C & $\begin{array}{l}\text { Un article } 20 \text { ayant commis une délin- } \\
\text { quance traditionnelle âgé de } 16 \text { ans et } \\
\text { plus et avec des antécédents à la Cour. }\end{array}$ & 668 & $12,67 \%$ \\
\hline $\mathbf{D}$ & $\begin{array}{l}\text { Un article } 20 \text { ayant commis une délin- } \\
\text { quance traditionnelle âgé de } 16 \text { ans et } \\
\text { plus et sans antécédents à la Cour. }\end{array}$ & 644 & $12,27 \%$ \\
\hline $\mathbf{E}$ & $\begin{array}{l}\text { Un écolier (article } 15 \text { ) dont les parents } \\
\text { vivent ensemble ayant commis une } \\
\text { délinquance nouvelle. }\end{array}$ & 392 & $7,43 \%$ \\
\hline $\mathbf{F}$ & $\begin{array}{l}\text { Un écolier (article 20) dont les parents } \\
\text { vivent ensemble et n'ayant pas commis } \\
\text { une délinquance nouvelle. }\end{array}$ & 44 &, $83 \%$ \\
\hline
\end{tabular}


TABLEAU 2 (suite)

Types de clients

\begin{tabular}{|c|c|c|c|}
\hline Type & Caractéristiques du type & $\begin{array}{l}\text { Nombre } \\
\text { de sujets }\end{array}$ & $\%$ \\
\hline$G$ & $\begin{array}{l}\text { Un écolier (article 20) n'ayant pas } \\
\text { commis une délinquance traditionnelle } \\
\text { et dont les parents ne vivent pas en- } \\
\text { semble. }\end{array}$ & 222 & $4,21 \%$ \\
\hline $\mathbf{H}$ & $\begin{array}{l}\text { Un non-écolier (article 20) ayant des } \\
\text { antécédents à la Cour et n'ayant pas } \\
\text { commis une délinquance tradition- } \\
\text { nelle. }\end{array}$ & 197 & $3,73 \%$ \\
\hline I & $\begin{array}{l}\text { Un non-écolier (article 20) sans anté- } \\
\text { cédents à la Cour et n'ayant pas com- } \\
\text { mis une délinquance traditionnelle. }\end{array}$ & 155 & $2,94 \%$ \\
\hline $\mathbf{J}$ & $\begin{array}{l}\text { Un article } 15 \text { qui est un garçon en } \\
\text { danger moral et âgé de } 1 \text { à } 15 \text { ans. }\end{array}$ & 651 & $12,35 \%$ \\
\hline $\mathbf{K}$ & $\begin{array}{l}\text { Un article } 15 \text { qui est une écolière en } \\
\text { danger moral et âgée de } 1 \text { à } 15 \text { ans. }\end{array}$ & 375 & $7,11 \%$ \\
\hline $\mathbf{L}$ & $\begin{array}{l}\text { Un article } 15 \text { qui n'est pas une éco- } \\
\text { lière mais elle est en danger moral et } \\
\text { âgée de } 1 \text { à } 15 \text { ans. }\end{array}$ & 77 & $1,46 \%$ \\
\hline $\mathbf{M}$ & $\begin{array}{l}\text { Un article } 15 \text { qui est un garçon de } \\
16 \text { ans et plus en danger moral. }\end{array}$ & 319 & $6,05 \%$ \\
\hline $\mathbf{N}$ & $\begin{array}{l}\text { Un article } 15 \text { qui est une fille de } \\
16 \text { ans et plus en danger moral. }\end{array}$ & 312 & $5,92 \%$ \\
\hline 0 & $\begin{array}{l}\text { Un article } 15 \text { ayant commis une dé- } \\
\text { linquance, c'est un écolier qui a com- } \\
\text { mis une délinquance nouvelle. }\end{array}$ & 122 & $2,31 \%$ \\
\hline $\mathbf{P}$ & $\begin{array}{l}\text { Un article } 15 \text { ayant commis une dé- } \\
\text { linquance; c'est un écolier. }\end{array}$ & 29 &, $55 \%$ \\
\hline $\mathbf{Q}$ & $\begin{array}{l}\text { Un article } 15 \text { ayant commis une dé- } \\
\text { linquance; c'est un non-écolier. }\end{array}$ & 73 & $1,38 \%$ \\
\hline $\mathbf{R}$ & $\begin{array}{l}\text { Un article } 15 \text { ayant commis une dé- } \\
\text { linquance ; c'est une fille de } 1 \text { à } 15 \text { ans. }\end{array}$ & 143 & $2,71 \%$ \\
\hline $\mathbf{s}$ & $\begin{array}{l}\text { Un article } 15 \text { ayant commis une dé- } \\
\text { linquance; c'est une fille de } 16 \text { ans } \\
\text { et plus. }\end{array}$ & 189 & $3,58 \%$ \\
\hline
\end{tabular}

quance et les cas de protection. Les premiers représentent au moins $60 \%$ de la clientèle et ce sont plus souvent des garçons ayant commis un délit criminel. Les seconds représentent $40 \%$ de la clientèle et ce sont plus souvent des enfants et de jeunes adolescents ayant des troubles de comportement ou un foyer problème. Cette conclusion est d'autant plus intéressante que Bouffard (1969) avait demandé aux agents de probation quel genre de cas devraient faire partie de leur case-load. L'analyse de ces 
FIGURE 1

Typologie de la clientète

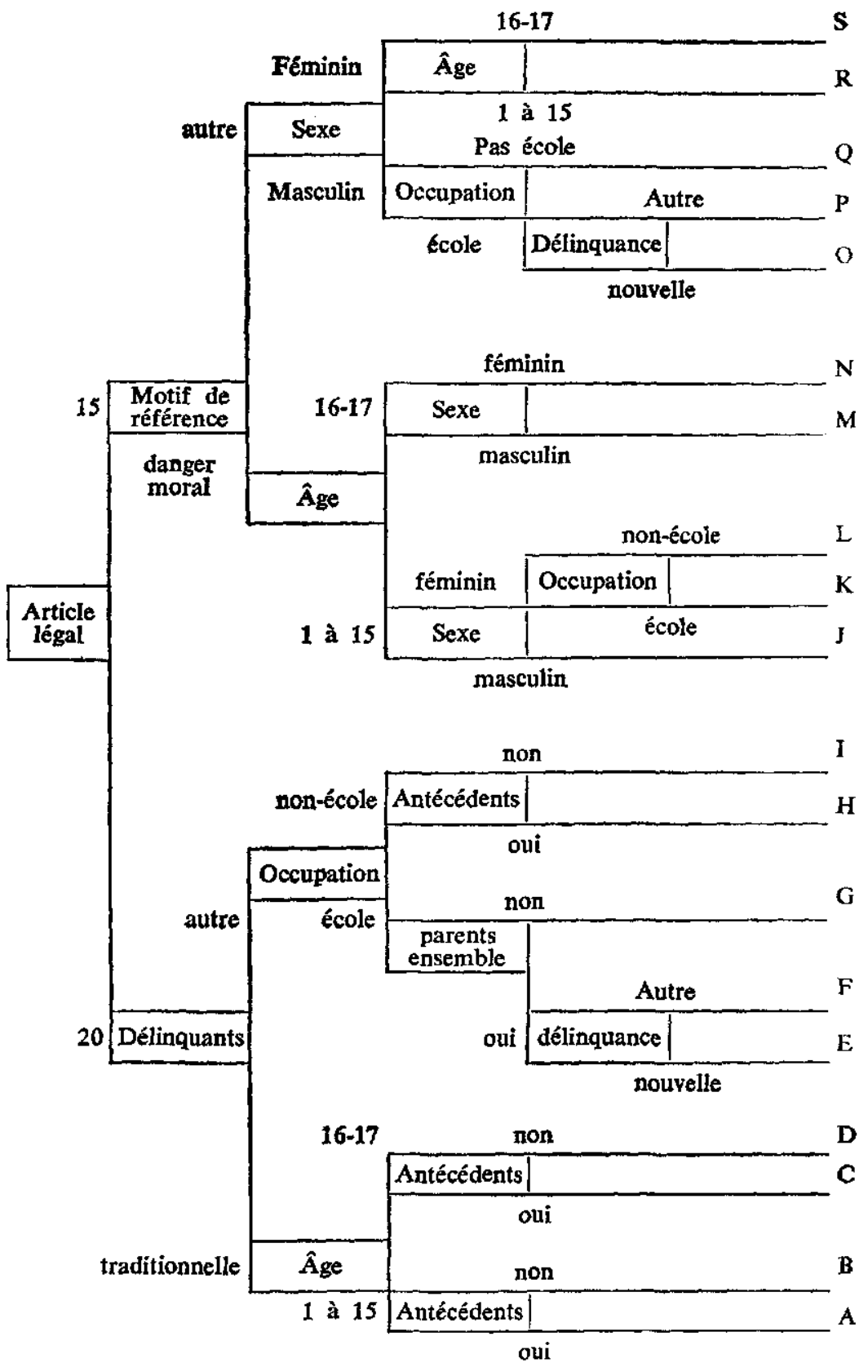


données (Cayen et al., 1970) a permis d'établir que pour les neuf cas de délinquance présentés, au moins $80 \%$ des agents les considèrent comme appartenant à leur case-load. Par ailleurs, pour les 15 cas considérés comme de protection, le consensus moyen est de $65 \%$ pour les définir comme cas propres à un Service de Probation.

\section{B. LE PERSONNEL}

Il n'existe pas de données systématiques et complètes sur le personnel du Service de Probation. En conséquence nous devrons nous référer aux données recueillies par diverses recherches, qui concernent plus ou moins l'ensemble des agents de Probation (Bouffard, 1967 ; Lippé, 1969 ; Brousseau et LeBlanc, 1974; Ministère des Affaires Sociales, 1972). Ces rapports nous permettront de situer l'identité sociale des agents de même que leur identité professionnelle; il sera aussi possible d'identifier des changements sur ces caractéristiques entre 1968 et 1973.

Les agents de probation sont plus souvent des hommes de 25 à 44 ans. En effet, Bouffard (1969) observait que 72\% des agents étaient de sexe masculin alors que Brousseau et LeBlanc (1974) notent une proportion de $57 \%$. Donc, il semble que l'on recrute de plus en plus d'agents de probation de sexe féminin. Cette situation peut apparaître anachronique quand l'on sait que la clientèle la plus nombreuse et la plus difficile est constituée de garçons de 16 et 17 ans.

Sur le plan de l'âge, on note une diminution de la moyenne d'âge qui passe de 36,8 ans (Bouffard, 1969) à 35 ans (Brousseau et LeBlanc, 1974). En cinq ans, la structure d'âge ne s'est pas radicalement modifiée car il y avait $82 \%$ d'agents de moins de 50 ans en 1969 (Lippé, 1969) et il y en a 80\% en 1970 (Brousseau et LeBlanc, 1974). Par ailleurs la proportion des moins de 35 ans est passée de $49 \%$ à $47 \%$ (Bouffard, 1969 ; Brousseau et LeBlanc, 1974). Somme toute, la structure d'âge des agents de probation demeure relativement stable et cette situation peut peutêtre s'expliquer par des facteurs de recrutement et de vieillissement du personnel.

Si le Service de probation compte de plus en plus d'agents de sexe féminin mais sans avoir rajeuni sa structure d'âge, il n'en demeure pas moins que le niveau de professionnalisation et d'expérience augmente. En effet, il y a plus de professionnels 
(diplôme universitaire), 8\% en 1968 (Bouffard, 1969), 10\% en 1970 (M.A.S., 1972) et $21 \%$ en 1973 (Brousseau et LeBlanc, 1974). Durant la même période les diplômés du collégial ont augmenté et il y a eu un effort pour favoriser la formation en cours d'emploi et l'éducation permanente. Sur le plan de l'expérience de travail, on observe que celle-ci s'accumule ; en effet $56 \%$ des agents (Bouffard, 1969) avaient 5 ans d'expérience ou moins tandis que maintenant cette proportion n'est que de $40 \%$ (Brousseau et LeBlanc, 1974); par contre si 9\% des agents avaient onze ans ou plus d'expérience en 1968 , il y en a maintenant $20 \%$ dans cette catégorie.

Au terme de cette description du personnel du Service de probation, il faut conclure que l'évolution de la qualité du personnel est certaine, la professionnalisation, par l'expérience et la formation, s'est accrue; par ailleurs, des questions demeurent quant à l'adéquacité du personnel en regard de la nature de la clientèle; en effet l'augmentation importante des agents féminins est-elle une solution quand la majorité des clients est composée de garçons et surtout de garçons de 16 et 17 ans.

\section{LES OPERATIONS}

Les opérations d'un Service dépendent de la définition de son champ de travail et celui du Service de probation n'a jamais été précisément défini. En effet, les premiers agents de probation assistaient tous les enfants qui comparaissaient devant le tribunal pour mineurs. Le volume de la clientèle a par la suite obligé à plus de sélectivité et l'on a opté pour les cas reconnus jeunes délinquants. Ainsi depuis 1970 on remet de plus en plus les cas de protection aux agences sociales. En 1972 (LeBlanc, 1972) on trouvait encore seulement $58 \%$ de cas de délinquance au Service de probation. Cette situation peut s'expliquer par le fait que le rapatriement des cas de protection est incomplet ou par l'utilisation différentielle que les juges font des articles légaux. A la lumière de cette situation on peut penser que la spécialisation et la professionnalisation du Service de probation sera freiner tant que son champ de travail ne sera pas clairement délimité.

La charge de travail des agents de probation est assez lourde ; ainsi en 1972 (LeBlanc, 1972) il y avait 5269 clients et 118 agents avec case load, soit une moyenne de 45 cas par agent. Le plus 
petit case load comptait 2 cas et le plus grand 165 cas (voir tableau 3).

Ces case-load de grandeur très différente, d'un agent de probation à l'autre, sont-ils spécialisés ? Du point de vue du sexe du client, $89 \%$ des case-load peuvent être qualifiés de spécialisés, dont $36 \%$ comprennent avant tout des filles et $52 \%$ sont composés en majorité de garçons. Par ailleurs, beaucoup moins de case-load sont composés de clients du même âge (58\%) : dont $2 \%$ qui comptent des clients de 1 à 9 ans, $14 \%$ comprennent avant tout des clients de 10 à 13 ans, $11 \%$ sont constitués de jeunes de $14-15$ ans et $31 \%$ de jeunes de 16 ans et plus.

TABLEAU 3

Grandeur du case-load

\begin{tabular}{|c|c|c|}
\hline & $\mathbf{N}$ & $\%$ \\
\hline 1 aे 5 & 4 & $3,38 \%$ \\
\hline $6-10$ & 7 & $5,93 \%$ \\
\hline $11-15$ & 3 & $2,54 \%$ \\
\hline $16-20$ & 11 & $9,32 \%$ \\
\hline $21-25$ & 9 & $7,62 \%$ \\
\hline $26-30$ & 7 & $5,93 \%$ \\
\hline $31-35$ & 12 & $10,16 \%$ \\
\hline $36-40$ & 8 & $6,77 \%$ \\
\hline $41-45$ & 11 & $9,32 \%$ \\
\hline $46-50$ & 7 & $5,93 \%$ \\
\hline $51 \cdot 55$ & 8 & $6,77 \%$ \\
\hline $56 \cdot 60$ & 6 & $5,08 \%$ \\
\hline $61 \cdot 65$ & 5 & $4,23 \%$ \\
\hline $66 \cdot 70$ & 1 &, $84 \%$ \\
\hline $71-75$ & 3 & $2,54 \%$ \\
\hline $76-80$ & 3 & $2,54 \%$ \\
\hline $81-85$ & 4 & $3,38 \%$ \\
\hline $86-90$ & 3 & $2,54 \%$ \\
\hline $91-95$ & 0 & \\
\hline $96-100$ & 0 & \\
\hline $101 * 105$ & 3 & $2,54 \%$ \\
\hline $121-125$ & 1 &, $84 \%$ \\
\hline $126-130$ & 1 &, $84 \%$ \\
\hline $161-165$ & 1 &, $84 \%$ \\
\hline Total & 118 & $100 \%$ \\
\hline
\end{tabular}


Après avoir décrit le champ et la charge de travail des agents de probation, nous pouvons aborder les opérations proprement dite, c'est-à-dire la durée de la prise en charge, la disposition des cas et la façon d'en arriver aux décisions. Pour ce qui est de la durée de la prise en charge, LeBlanc (1972) établit que la clientèle nouvelle compte pour environ un quart du caseloard; de plus le client est en général servi rapidement car dans $65 \%$ des cas, il y a moins d'une semaine entre la date de prise en charge et la date de la première mesure. La durée de la prise en charge moyenne est de 51 semaines, la plupart des cas le sont pour moins de six mois et un tiers pour plus d'un an. Ainsi une probation durera en moyenne 37 semaines, un placement en foyer 55 semaines (ceci indépendamment des transferts d'un foyer à l'autre) et une institutionnalisation 74 semaines.

Quant à la disposition des cas, il faut noter (LeBlanc, 1972) qu'un tiers des clients étaient en observation comme première mesure prise, $29 \%$ étaient en probation, $18 \%$ en foyer supplétif et $20 \%$ en institution. Il a aussi été démontré que le choix de cette première mesure dépend de l'âge, du sexe, du délit et des antécédents du sujet; ainsi, les cas placés directement en institution seront des garçons de 16-17 ans, des récidivistes ayant volé; par contre, celui qui arrive au Service pour un premier délit, qui somme toute est bénin, ceci vers 13-14 ans, sera plus facilement placé en foyer supplétif ou en observation. Quant à la mesure finale, il faut noter que le placement en foyer diminue de moitié $(18 \%$ à $9 \%)$, la probation est utilisée pour $34 \%$ des cas au lieu de $29 \%$ et $35 \%$ des cas sont institutionnalisés (dans 112 institutions) plutôt que $20 \%$. Ainsi $30 \%$ des cas placés initialement en foyer ou en probation sont par la suite institutionnalisés.

En somme, le Service de probation se caractérise par un roulement continu de $25 \%$ des cas, $35 \%$ des cas occasionnent peu de travail à l'agent (sauf dans la phase initiale) car ils sont en institution pour environ un an et demi et seulement $40 \%$ des cas demandent une surveillance constante et une intervention spécifique. Finalement, il convient de noter que moins de $10 \%$ des cas sont transférés d'un agent à l'autre.

Nous avons vu que les trois quarts de la clientèle du Service de probation est soit en institution soit en liberté surveillée (dans la famille ou dans un autre foyer), comment les agents de pro- 
bation arrivent-ils à faire le choix de la mesure à prendre ? Dans un article récent, Brousseau et LeBlanc (1974) répondent à cette question et ils formulent la conclusion générale suivante : l'analyse des données démontre une absence d'uniformité minimum dans la façon de prendre une décision et dans la recommandation qui s'ensuit.

En effet, les agents de probation utilisent entre 3 et 42 informations soit en moyenne 21 informations. La différence dans le nombre d'informations utilisées est donc très importante, à la fois en elle-même et par rapport à d'autres études comparables (on y utilisait en moyenne un quart des informations par rapport à la moitié ici). Cette constatation peut s'expliquer par la nature du problème, la délinquance juvénile par rapport à la criminalité adulte, par la diversité de formation et d'attitude des agents de probation et par le climat d'incertitude qui marque le service actuellement. Néanmoins, la diversité pour un même cas est telle, que l'on doit se demander si l'individualisation de la prise de décision ne pénalise pas les clients.

Cette interrogation demeure lorsque l'on analyse la qualité de l'information utilisée. En effet, à chaque choix, chaque étape du processus de la prise de décision, la diversité des informations choisies tend à approcher la moitié des informations disponibles. Si l'uniformité est loin d'un consensus minimal sur le type d'information utilisé, il en est de même pour les stratégies de cueillette des informations. Ainsi la cueillette des informations s'étale sur quatre phases : démarrage, consolidation, approfondissement et conclusion. À mesure que l'on passe d'une phase à l'autre, le degré de spécificité des informations tend à devenir fonction du hasard. Ainsi à la quatrième phase, la conclusion, aucune information n'est sélectionnée par plus de $5 \%$ des agents de probation pour mineurs. A cette diversité dans la qualité de l'information choisie, s'ajoute une grande variété de stratégies de cueillette des informations.

Si l'on analyse la recommandation faite, on note une diversité aussi grande que celle pour la prise de décision. En effet, même si le cas présenté était considéré comme typique d'une institution (il y avait même séjourné) il n'en demeure pas moins que la moitié des agents de probation a opté pour une mesure en liberté surveillée et que l'autre moitié a opté pour une mesure d'institutionnalisation. Seulement $17 \%$ des agents ont recommandé une 
mesure en conformité avec celle déjà adoptée pour le cas. Ces résultats doivent être considérés à la lumière du fait que les agents étaient confiants dans la recommandation et qu'ils disent avoir pris la décision facilement. Comment expliquer, pour un même cas, cette variabilité dans la recommandation ?

Si l'on étudie maintenant le processus décisionnel (Brousseau et LeBlanc, 1973) on observe que d'une façon générale $(2 / 3$ des cas) quand un placement institutionnel est envisagé, l'évaluation clinique est demandée et il semble bien que c'est celle-ci qui vient fixer l'orientation définitive du cas, puisque l'agent suit la recommandation de l'évaluation clinique dans la presque totalité des cas. Par ailleurs, il est courant de discuter du cas avec les cliniciens, en plus d'analyser leur rapport.

De plus, l'agent discute de la situation avec le sujet lui-même ( $5 / 6$ des cas), avec le juge et les parents (4/6 des cas) et avec des supérieurs, confrères et / ou représentants de l'institution ou d'autres ressources (2/6 des cas). Les résultats rapportés nous permettent de conclure à une utilisation inégale de l'évaluation clinique et de la discussion de cas. On peut expliquer cette constatation par un manque de ressources à cet effet, mais on peut aussi penser que les évaluations cliniques sont de moins en moins nécessaires compte tenu du degré de professionnalisation croissant des agents de probation.

En terminant cette section sur les opérations au Service de probation, il faut signaler que la description ci-dessus décrit l'ensemble mais que les variations d'un service local à l'autre (il y en a 14) sont très importantes (LeBlanc, 1972; Brousseau et LeBlanc, 1973).

\section{L'AVENIR DE LA PROBATION POUR MINEURS}

Terminer cet article en abordant le thème de l'avenir de la probation pour mineurs, c'est dégager les grandes tendances dans l'évolution du Service et de là, lui fixer des points d'artivée. C'est signaler les gestes concrets que devraient poser les agents de probation, la direction du Service et la direction du ministère, pour faire passer le Service de probation de l'état d'une mesure carrefour à l'état d'une mesure spécifique et complémentaire aux autres.

Initialement, la probation était un service à la cour, consultation aux comparutions, évaluation des cas, placement en foyer 
nourricier, placement en institution et une mesure de liberté surveillée (surveillance et traitement en milieu ouvert). Depuis un processus s'est engagé qui permettra de spécifier le rôle de la probation : les cas de placement en foyer nourricier ont été remis aux agences sociales et on parle de plus en plus de remettre au CSS les placements en institution. En conséquence de ces mesures, les clients de 14 à 18 ans, les garçons et les cas de délinquance prennent une place prépondérante parmi la clientèle du Service de probation.

Parallèlement à ces mesures administratives et de la spécification de la clientèle qui s'en suit, les attentes de rôles des agents de probation (Bouffard, 1969) exprimaient clairement un intérêt pour les fonctions de traitement, d'information-conseil auprès des clients et de consultation auprès du tribunal. Durant cette période de transformation, la formation et l'expérience des agents se sont nettement améliorées.

La lecture de ces lignes d'évolution du Service de probation nous amène à suggérer que soit terminé définitivement le transfert des cas de placement en foyer nourricier aux agences sociales et que les comités d'admission des CSS aux Centres d'accueil jouent vraiment leur rôle et prennent en charge le placement en institution et la supervision des cas. Ces mesures prises, le Service de probation verra son case-load diminuer d'environ $40 \%$ et il pourra se concentrer sur la liberté surveillée. Par ailleurs, on devrait distinguer l'évaluation clinique des cas et la consultation auprès du tribunal de la mesure particulière de liberté surveillée, qui suppose surveillance et traitement. Il faut, auprès du tribunal, un service complet de diagnostic et indépendant de la probation. Cette division du travail permettra de bien distinguer des rôles souvent conflictuels celui d'agent de traitement qui met l'accent sur le client et ses besoins et celui d'agent de diagnostic qui doit avoir à l'esprit la dangerosité sociale du sujet et qui représente, d'une certaine manière, l'autorité du tribunal.

Le processus engagé de centration du Service de probation sur la mesure spécifique de liberté surveillée pourra se réaliser si une mentalité de complémentarité entre les ressources prend le pas sur une mentalité d'impérialisme et dans la mesure où chaque ressource sera consciente que son personnel et son action doivent correspondre aux objectifs qu'elle vise. 


\section{BIBLIOGRAPHIE}

BOUFFARD, D. (1969) : Etude du rôle de l'officier de probation, Montréal, thèse de maîtrise inédite en criminologie, Université de Montréal.

BROUSSEAU, G. et LEBLANC, M. (1973) : la Référence au Mont Saint Antoine : Processus décisionnel, Montréal, Groupe de recherche sur l'inadaptation juvénile.

BROUSSEAU, G, et LEBLANC, M. (1974) : La prise de décision et la recommandation de l'agent de probation pour mineurs s, Revue Canadienne de Criminologie, 16, 4, p. 373-392.

CAYEN, G., LAMARCHE, P. et BUISSIERES, C. (1970) : Validation d'hypothèses en fonction du consensus du rôle des officiers de probation, séminaire inédit, Département de criminologie.

LEBLANC, M. (1971) : Méthodes quantitatives : analyse des données, Montréal, Département de criminologie.

LEBLANC, M. (1972) : la Probation juvénile au Québec : inventaire du case-load, Montréal, Groupe de recherche sur l'inadaptation juvénile.

LIPPE, M. (1969) : Etude du consensus entre les juges et les officiers de probation des C.B.E.S. du Québec sur le rôle de l'O.P. dans l'administration de la justice pour mineurs, Montréal, thèse de maîtrise inédite en Service social, Université de Montréal.

MINISTERE DES AFFAIRES SOCLALES (1972) : les Services d l'enfance inadaptée : le personnel, la répartition et le coût des Services, Québec, Gouvernement du Québec. 